\title{
MOBILITY FOR HEALTH: DESIGNING OPEN PARKS TO ENCOURAGE PHYSICAL ACTIVITY
}

\author{
CHLOE KADI \& ANDREAS SAVVIDES \\ University of Cyprus, Cyprus
}

\begin{abstract}
The objective of this study is to investigate how the planning and design of open parks within neighbourhoods and communities can promote physical activity in order to enhance the health of the local population. An extensive literature review was conducted for studies regarding the relationship between health and physical activity and on the park characteristics that can promote physical activity among people. The findings of the literature review were then compared and analysed in order to identify the main characteristics of urban parks that can promote physical activity and enhance public health. In order to find out how the characteristics identified in the literature were applied in real life, an analysis of three existing parks in three different countries was conducted. The parks, apart from their geographical location, also vary in size and layout. The parks were chosen because they are urban open parks and they include facilities for physical activity.

Keywords: health, public health, well-being, physical activity, leisure-time physical activity, urban planning, active living behaviour, open parks.
\end{abstract}

\section{INTRODUCTION}

How can the planning and design of open parks within neighbourhoods and communities promote physical activity in order to enhance the health of the people?

The aim is to investigate the parameters that should be taken in account in the planning and design of open parks within neighbourhoods/communities in order to enhance and ameliorate the quality of life of people in the community. Due to time and scope limitations, the current research investigates the features and characteristics that a park must provide in order to encourage physical activity for its users.

\subsection{Rationale}

Studies have shown that physical activity (PA) can reduce the probability of earlier mortality by decreasing such diseases as heart disease, diabetes, high blood pressure, cancer, depression, anxiety, and obesity while building up the body and ensuring the health of human bone structure, skeletal joints and muscular disposition [1], [2].

Unfortunately, PA levels have declined over recent decades, with a shift towards sedentary lifestyle [3]. Today, physical inactivity and unhealthy diet are second only to tobacco as the main causes of premature death [4]. Reversing this trend could confer considerable population health benefits [3].

Literature indicates two main factors for the shift of people towards sedentary lifestyle: The personal factors related mainly to gender, race, age, ethnicity, socioeconomic status and religion and need a behavioural change and factors arising from the urban design of neighbourhoods/communities and cities [1]-[3]. Targeted education programs can affect behavioural change of the individuals. At the same time, there must be changes and interventions at the neighbourhood scale of the community to make it easier for people to be physically active. Such interventions include, among others, design that promotes active transport, designing neighbourhoods so as to include a mixture of facilities with related 
infrastructure for a multitude of activities for exercise and leisure, such as outdoor exercise equipment and play areas connected by various park trails [1].

Research into this topic indicates that approaches utilizing evidence-based tools to inform decisions and plans at the architectural and urban design scale can also make a positive contribution to the inclusion of physical activities for health [4]. Such strategies as those prescribed by proponents of Active Design - that is designing urban spaces in such a way so as to promote an active lifestyle improve not only the physical condition of city dwellers, but also the physical qualities and maintenance of open spaces in the cities, such as parks, as the more frequent interactions of city dwellers with these facilities, leads to a demand for higher quality environments.

Such measures are those that alter people's decisions about how to handle everyday trips for work of access to services and amenities by having them decide against the use of mechanical means of transport and choose to take their bicycle or to walk to their destination or to choose to climb a stair rather than taking the elevator and this advice is proposed for people of all ages, regardless of gender and accounting for various conditions of physical mobility, as long as appropriate infrastructural support is provided [4].

In fact, due to sedentary jobs and lifestyle and the increasing dependence on motorised transport, the opportunity for a more active lifestyle, both for the purpose of everyday errands as well as for purposes of leisure, has to be sought at every opportunity and to always present a viable and healthier alternative [1], [4].

Whether engaging green or more recently green and blue infrastructures - that take into account the aquatic parameter of planning for green spaces and parks - these amenities need to find physical correspondence to major corridors of mobility, commercial and civic centres and nodes of education and culture [5].

Due to time and scope limitations, the current research investigates the features and characteristics that a park in a neighbourhood must provide in order to encourage physical activity for its users.

Local parks, offer the opportunity for physical activity at low or no cost [1]. Yet, it is recognised that park environmental characteristics such as features, access, condition, aesthetics, safety and policies are most related to physical activity [1].

Study objectives:

- Investigate whether physical activity can positively influence public health.

- Investigate how urban design can encourage physical activity and promote an active lifestyle for people with specific focus on public/communal spaces and open parks.

- Investigate the core benefits of parks to park users.

- Identify the park characteristics that encourage people to visit parks and promote physical activity.

The key questions to be answered:

- What is the definition of "Health" and "Public health"?

- Does physical activity positively influence health?

- How can urban design and built environment encourage physical activity?

- What are the benefits of open parks on peoples' health?

- What are the main characteristics of park design and layout which can promote physical activity and public health? Investigate how urban design can encourage 


\section{LITERATURE REVIEW}

According to the World Health Organization [6], health is "a state of complete physical, mental and social well-being and not merely the absence of disease or infirmity". Public Health is defined as "the science and art of promoting and protecting health and well-being, preventing ill-health and prolonging life through the organized efforts of society" [7].

\subsection{Physical activity and health}

Physical Activity (PA) and health related to the design and building of parks are directly linked to economic performance and economic indicators that on one show property values increasing with closer proximity to these green spaces but also poverty levels decreasing, resulting in more resilient and socially cohesive communities across many cultures and mentalities [8]. The constant goal of such international bodies is to make people and especially city dwellers who may perhaps lead more sedentary lives to institute lifestyle changes by adopting pastimes directly associated with the presence and close proximity of parks to work and home [5] that improve the quality of life and may even extent its span [7] as a result of improved health.

WHO's PA strategy for the European Region for the period 2016-2025 targets specifically prevalent type of sedentary lifestyles mentioned above with the aim of achieving real change through a decrease of indicators associated with medical conditions - not including communicable diseases - that are directly attributed to lact of physical activity. These kinds of ailments - that is non-communicable diseases (NCDs) - are some of the main causes of death in Europe [5].

In the USA alone, an estimated 200,000 to 300,000 deaths are attributed to a distinct lack of physical activity [2]. Action on the top seven risk factors, namely: high blood pressure, high cholesterol, high blood glucose, excess weight, physical inactivity, tobacco smoking, and alcohol abuse - cause a reduction in disability-adjusted life-years (DALYs) lost by nearly $60 \%$ in the WHO European Region and $45 \%$ in high-income European countries [8].

WHO defines PA as an important foundation of health throughout life. Apart from its known health benefits regarding NCDs, it also has positive effects on mental health by reducing stress reactions, such as anxiety and depression and by possibly delaying the effects of Alzheimer's disease and other forms of dementia. Furthermore, PA is fundamental to achieving energy balance and weight control [5]. Among older people, PA helps to maintain health, agility and functional independence and to enhance social participation. It may also help in balance and stability and in assisting in chronic disease rehabilitation, becoming a critical component of a healthy life [5].

Physical activity can be effective at all phases of chronic disease management, from primordial prevention (prevention of risk factors) through treatment and rehabilitation. The prevention of chronic diseases results to the improvement of quality of life and the reduction of health care costs [9]. Recommended guidelines for physical activity encompass four components: frequency, time or duration, type and intensity of physical activity [1].

There are different forms, kinds and levels of intensity of PA. These include fundamental movement skills, active play, leisure activities, such as walking, dancing, hiking and biking, sports and structured exercise [5]. Sallis et al. [9] classifies physical activity into four domains of life that describe how people spend their time: leisure/recreation/exercise, occupation/school, transportation, and household. The four domains are relevant to and driven by different built environment features and policies.

WHO [5] recommends that adults and older people undertake at least 150 minutes of moderate-intensity aerobic physical activity each week. Children and young people should 
accumulate at least 60 minutes of moderate to vigorous-intensity physical activity every day. However, it is recognised that a small amount of PA is better than none [5].

Moderate-intensity aerobic physical activity is equivalent to brisk walk. Vigorousintensity physical activity is exemplified by jogging [10]. To promote and maintain good health and physical independence, adults will benefit from performing activities that maintain or increase muscular strength and endurance for a minimum of two days each week. (It is recommended that 8-10 exercises be performed on two or more non-consecutive days each week using the major muscle groups). To maximize strength development, climbing stairs is appropriate, among other activities [10].

\subsection{The effect of the built environment on physical activity and public health}

Built environments are all places built or designed by humans, including buildings, grounds around buildings, community facilities, transportation infrastructure, parks and trails [9]. According to Troped [11] the built environment includes the man-made surroundings that provide settings that may be used or appropriated or accessed for purposes of engaging in physical activity, such as neighbourhoods, streets, public transportation systems, commercial centres, schools, parks, trails and other outdoor recreational spaces. The design of the built environment can have a crucial and positive influence on improving public health [4].

Characteristics of built environments, including the transportation systems, have been related to rates of chronic disease, mental health and risk factors [9], [12]. The degree of accessibility designed in the built environment can also encourage persons with disabilities to be physically active and to be socially integrated into their community [12]. Physical activity can be fostered by designing spaces and streets that encourage walking, cycling, and other forms of active transportation [4] and by creating facilities such as trails, swimming pools, parks, courts, greenways, soccer fields, picnic areas, open spaces and playgrounds [1], [9] that allow people to engage in leisurely activities that satisfy the PA requirement.

Research on the relationship between the built environment and health has largely focused on housing, transportation, and neighbourhood characteristics [13].

Pate et al. [14] indicates that the environment often presents important barriers to participation in physical activity, including a lack of bicycle trails and walking paths away from traffic, inclement weather, and unsafe neighbourhoods. Other studies have consistently shown an association between a deteriorated physical environment and higher rates of crime, making neighbourhoods less safe for walking and in some cases resulting in greater social isolation [13].

The implications and significance of built environment to promote physical activity and public health are evident throughout the literature. The scope of this study has been limited to the effects of park design to physical activity alone. For completeness, a brief summary of the role of the built environment on public health and of other elements of the built environment that can promote physical activity can be found below.

\section{PARKS AND PUBLIC HEALTH}

Local parks are close-to-home facilities and services available at low or no cost. According to studies [15], local parks and organized recreation programs and facilities are appreciated by people and their communities are a result of extracting specific benefits. These benefits can take many different forms including,

- Personal benefits, which are pertinent to an individual's health and physiological and mental wellbeing, such as finding a good balance between active and sedentary past 
times that may be addressed by exercise one hand and relaxation and leisure in the context of natural environments on the other.

- Environmental benefits, which look at the health of entire eco systems and look to find balance in nature between the flora and fauna and the human presence.

- Social benefits, which look not at the individual but rather as the collective and enhance community resilience and acceptance by providing places for the community to cohabit and interact and is characterized by diverse locales for a diverse community.

- Economic benefits, which look at reciprocal and multiplying effects that may be realized from closeness to the park facilities but also from complementary uses that may enhance commercial activities as venues of exchange that add value to the broader area, like availability, bringing business activity to community, influence on property values

- Cultural benefits, which look at parks as not only venues for relaxation, leisure or physical activity but also as spaces for education and for the production of culture by hosting a diverse range of activities that transform them to outdoor classrooms.

Local parks are also critical in preserving the natural resources for communities, protecting open space and connecting children to nature. They also contribute to improving the overall health and wellness of the community by promoting social equity [15] by acting as social levellers through the programs that may be offered that are universally accessible and that appeal and bring together very diverse groups of people activity.

Moreover, the National Recreation and Park Association [16] includes to the above benefits, those that play a key role in the physical development of youths and young adults and to their mental development and physical wellbeing as well. They can achieve these by hosting organized activities that develop social skills through interactive and collaborative activities in nature, that teach them how to analyse and resolve challenges individually and collectively with a view to becoming more responsible and informed citizens of their community. In Fig. 1, the lower section of the model demonstrates the factors that influence the frequency of use and non-use of a park with associated benefits.

\section{Outcomes}

Benefits of parks and park usage

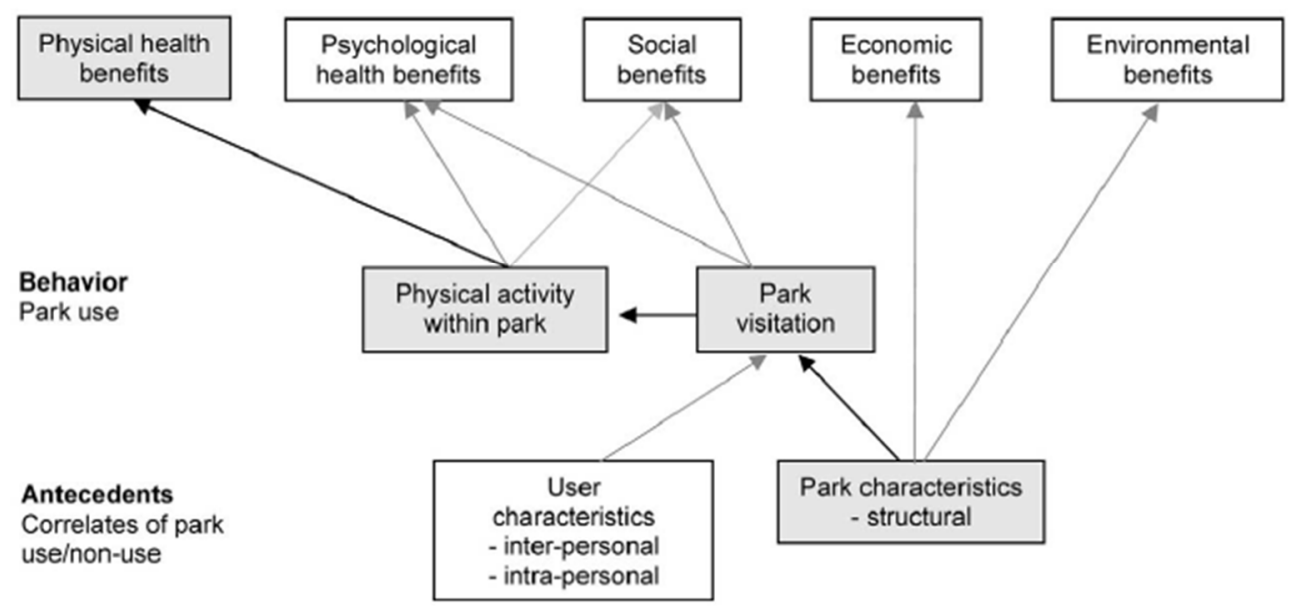

Figure 1: Relationship between park and physical activity [1]. 


\subsection{Physical health benefits}

Parks that are conveniently located are associated with physical activity from which are derived health benefits that are related to enjoying the cinematographic sensation of engaging natural scenery while engaging in a more active lifestyle [1], [13] encompassing activities in beautiful natural settings that elevate the human spirit.

\subsection{Psychological health benefits}

As mentioned above, the human spirit may be uplifted at the same time that one benefits from physical activity if this happens in a natural setting conducive to the amelioration of the human psyche, emotions and mental stability. Even the simple act of looking out a window as such places as community parks may be an uplifting experience. Other studies found that park users were in a better mood and reported lower levels of anxiety, stress and sadness after visiting parks [1]. In such settings that allow the individual to "get their mind of things" by engaging them both physically and emotionally has led to the reduction of depressive feelings or even to the betterment of an individual personal outlook towards a more positive approach to life's challenges [1], [5], [13].

\subsection{Social benefits}

Community pride may also be enhanced by the presence of well-kept parks especially in cases where the creation and maintenance of said parks turns out to be a collective activity that allows people to share this common asset through participatory and collaborative efforts. Social cohesion is strengthened by the taking place of common social activities and the setting of common social goals and in seeing them through by turning into settings where healthy behaviour (such as physical activity) is modelled [1] and where crime, aggression and violence are inhibited and social interaction among individuals promoted [1], [3], [15].

\subsection{Economic benefits}

Several studies pointed out that proximity to a park or reservoir - that is green and blue infrastructures, whether natural or manmade - has been shown to have a positive correlation to the real estate value of adjacent properties [1], [15].

\subsection{Environmental benefits}

The amassed environmental benefits that may be attributed to the increased presence of trees in parks has also been shown to combat the negative consequences of emission, such as those from automobile exhaust fumes in urban areas, by reducing air pollution and by providing shading and cooling [1], [15] and shaping the microclimate.

\section{PARK CHARACTERISTICS AND RELATIONSHIP TO PHYSICAL ACTIVITY}

With industrialization in the 19th century many of these nations and cities saw the first large scale appearance of organized open spaces and parks - some of them themed - with a view to providing open air spaces for congregations and common events thereby providing a healthier outlook from the severely dense and crammed conditions that prevailed at their work places and their places of habitation. These new spaces provided a proximity to nature and the natural, the trees were seen as filtering the polluted air and the healthier, cleaner 
surroundings were seen as countering what was perceived as the moral decay that characterized inner cities [17].

The role of parks continues to be as important today and not only for the reasons that brought them about in the past. In more recent times parks are also seen as places of leisure and recreation and learning, lending themselves to a variety of activities such as sports and markets and leisure and outdoors concerts and a variety of other cultural and commercial activities that appeal to a diverse community regardless of age, gender, condition of personal mobility or ethnic backgrounds as stated by Healthy People 2020 and the Institute of Medicine and cited accordingly by Sallis [9] and Bedimo-Rung [1].

It is therefore important for parks to have an inherent flexibility in the types of activity they may host and to cater to both the body and the soul, so to speak, by addressing the negative effects on human physiology and human psychology that may result from stressful situations experienced by city dwellers and to have also a calming effect on the community. These behaviours need to be examined and translated into reciprocal physical planning and design strategies for parks so as to become part of guidelines and bylaws governing urban design and community development initiatives by municipalities and communities [1]. In fact there are significant variations in park and outdoor recreation behaviours based on a number of demographic or social characteristics such as age, gender, race/ethnicity, socioeconomic status physical, cognitive or sensory ability and residential location [1], [3], [17], [18], but in this case they are not the focus of the current study. What is more pertinent are the environmental characteristics, which according to Bedimo-Rung [1], are comprised of six conceptual areas that operate through four geographic areas to encourage physical activity within parks, as demonstrated in Fig. 2.

\section{CONCLUDING THOUGHTS}

There is a vast amount of research studying the role of urban design towards a more sedentary lifestyle, which is associated with lower levels of physical activity. It is also widely recognised that increasing the levels of physical activity to 30 minutes a day may positively influence the health of people, especially in relation to non-communicable diseases (physical and mental) associated with insufficient activity levels, which are identified as a major reason for premature deaths. Research on the connections between the built environment and health has largely focused on housing, transportation, and neighbourhood characteristics. The current study was focused on the main characteristics of park design and layout which can promote physical activity and public health.

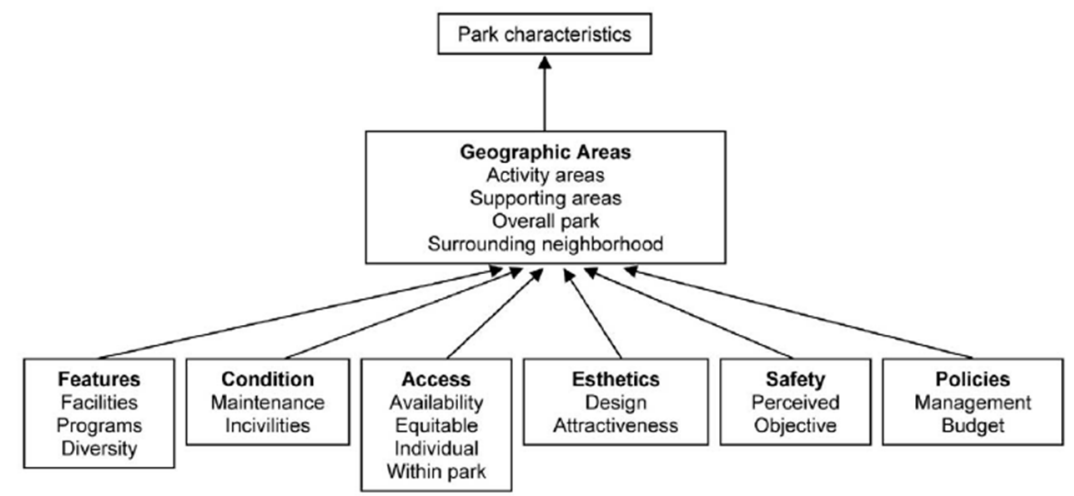

Figure 2: Environmental classification of park attributes [1]. 
The investigation has also verified what holds true for many a public infrastructure that is that people may be reluctant to visit a park if its maintenance and that of all related park facilities and equipment is poor or when concerns regarding crime and safety within the park, arise. Applying measures that ensure safety and good condition in open parks is essential for park users. The size of the park was also mentioned in the literature as an important factor for attracting people. The design of trails connecting smaller parks with limited features within a city may also encourage people to visit parks for more physical activity.

A number of environmental characteristics of open parks which can promote more physical activity have been identified in the literature and spelled out in the recommendations. Well-designed open parks in close distance to homes, which are easily accessible, offer a range of activities and supporting areas for all ages and provide an overall appealing environment. They provide city dwellers with options that offer them essential physical, mental and social benefits, in general, as well as more specific benefits for youth and young adults, as spelled out in the preceding text. Moreover, if one now considers the stress that has been placed on communities as a result of the COVID-19 restrictions regarding social distancing, parks may provide a flexible and evocative setting for many cultural and leisure but also commercial and even business-related activities that may occur outdoors.

Well-designed open parks are important community resources. Future research may focus on further environmental characteristics for open parks located in countries with hot climates. The association between physical activity levels in these countries and the specific park characteristics mentioned in the current study could be investigated and analysed.

\section{ACKNOWLEDGEMENT}

Our thanks to the Department of Public Works of the Republic of Cyprus.

\section{REFERENCES}

[1] Bedimo-Rung, A. et al., The significance of parks to physical activity and public health. A conceptual model. American Journal of Preventive Medicine, 28, pp. 159$165,2005$.

[2] Heath, G. et al., The effectiveness of urban design and land use and transport policies and practices to increase physical activity: A systematic review. Journal of Physical Activity and Health, 3(1), pp. 55-76, 2006.

[3] Lee, A.C.K. \& Maheswaran, R., The health benefits of urban green spaces: A review of the evidence. Journal of Public Health, 33(2), pp. 212-222, 2010.

[4] City of New York, Active design guidelines. Promoting physical activity and health in design, 2010. www1.nyc.gov/site/planning/plans/active-design-guidelines/activedesign-guidelines.page. Accessed on: 27 Jan. 2017.

[5] WHO, Physical activity strategy for the WHO European Region 2016-2025, 2015. www.euro.who.int/_data/assets/pdf_file/0010/282961/65wd09e_PhysicalActivity Strategy_150474.pdf. Accessed on: 21 Jan. 2017.

[6] World Health Organization Constitution, 1948. www.who.int/governance/eb/who_ constitution_en.pdf. Accessed on: 18 Jan. 2017.

[7] UK's Faculty of Public Health, What is public health? 2010. www.fph.org.uk/what is_public_health. Accessed 18 Jan. 2017.

[8] Jakab, Z., Designing the road to better health and well-being in Europe. Paper presented at the 14th European Health Forum, Bad Hofgastein, Austria, 2011. www.euro.who.int/_data/assets/pdf_file/0003/152184/RD_Dastein_speech_ wellbeing_07Oct.pdf. Accessed on: $\overline{18}$ Jan. 2017. 
[9] Sallis, J. et al., The role of built environments in physical activity, obesity, and CVD, NIH Public Access Author Manuscript, 2012.

[10] Haskell, et al., Physical activity and public health, Updated recommendation for adults from the American College of Sports Medicine and the American Heart Association, 2007. http://circ.ahajournals.org. Accessed: 14 Jan. 2017.

[11] Troped, P., The Power of Trails for Promoting Physical Activity in Communities. A Research Brief, Active Living Research, a National Program of the Robert Wood Johnson Foundation: Princeton, 2011.

[12] Dannenberg, A. et al., The impact of community design and land-use choices on public health: A scientific research agenda. American Journal of Public Health, 93(9), pp. $1500-1508,2003$

[13] Srinivasan, et al., Creating healthy communities, healthy homes healthy people. American Journal of Public Health, 93(7), pp. 1446-1450, 2003.

[14] Pate, R. et al., Physical activity and public health - A recommendation from the centers for disease control and prevention and the American college of sports medicine. JAMA, 273, pp. 402-407, 1995.

[15] Mowen, A.J., Graefe, A.R., Barrett, A.G., Roth, K. \& Godbey, G.C., Americans' broad-based support for local recreation and park services: Results from a nationwide study. National Recreation and Park Association: Ashburn, 2016.

[16] National Recreation and Park Association, Summary of research papers: The key benefits, 2010. www.nrpa.org/uploadedFiles/nrpa.org/Publications_and_Research/ Research/Papers/Synopsis-of-Research-Papers.pdf. Accessed on: 10 Mar. 2017.

[17] Giles-Corti, B. et al., Increasing walking: How important is distance to, attractiveness, and size of public open space? American Journal of Preventive Medicine, 28(2), pp. 169-176, 2005.

[18] National Physical Activity Plan Alliance. Physical Activity Plan, 2016. http://physicalactivityplan.org/docs/2016NPAP_Finalforwebsite.pdf. Accessed on: 9 Feb. 2017. 